\title{
Normal Development and Post-Traumatic Plasticity of Corticospinal Neurons in Rats
}

\author{
Constance J. D’Amato and Samuel P. Hicks ${ }^{1}$ \\ Defartuent of Pathology. Unizersity of Michigan Medical Center, \\ Ann Arbor, Michigan 48109 \\ Recived Warch 7,1978
}

\begin{abstract}
Corticospinal (CS) neurons projecting to the spinal cord in the adult rat, identified by retrograde axonal transport of horseradish peroxidase (HRP), formed a caudal band in areas 3,4 , and 6 and a rostral band in area 10 , separated by a gap. In the infant the gap was filled with CS neurons. The problem: What happened to the transient infant neurons as the mantle expanded, and would they persist if other CS neurons were destroyed in infancy? Identification of CS neurons by HRP and measurements of the growth of the mantle and cortical areas 3, 4, and 6 showed that CS neurons were scattered widely in the cortex as well as in the gap and future bands at 2 to 10 days. By about 2 weeks, CS neurons labeled from the cervical cord were limited to the "adult" bands. The greatest mantle expansion postnatally was in the occipital and bregma regions, including the anterior, but not the posterior, part of areas 3,4 , and 6 . Thus, expansion of the mantle, growth of areas 3,4, and 6, and axonal growth of transient and permanent CS neurons did not parallel each other closely. When one or both caudal band regions were ablated at 5,7 , or 10 days, the gap CS neurons persisted bilaterally to adult life. No necrosis of layer $V$ neurons was observed between 10 days and 2 weeks. It was assumed that the gap neurons and other extraneous CS neurons generated exploratory axons which normally disappeared, but when caudal band neurons were destroyed the transient axons attempted to fill the pathway.
\end{abstract}

\section{INTRODUCTION}

Previous studies (8) showed that corticospinal (CS) neurons which project to the spinal cord in the mature rat are arranged in two bands in

${ }^{1}$ This research was supported by U.S. Public Health Service grant NS 10531.

Abbreviations: CS-corticospinal; HRP-horseradish peroxidase; $\mathrm{Cl}$-first cervical level; L1-first lumbar level. 
the dorsal cortex separated by a gap. The larger caudal band corresponded substantially to Krieg's (10) cytoarchitectural areas 3, 4, and 6, and the rostral band lay in the anterior part of area 10 . The neurons were located by labeling with horseradish peroxidase (HRP) introduced to the cut ends of their axons at different levels of the spinal cord. The rostral band could be labeled only from cervical levels; neurons in the caudal band were labeled from cervical or lumbar levels, the neurons projecting to these two levels being generally intermixed through the band. The gap between the bands in mature rats, $2 \mathrm{~mm}$ or more in length, contained very few or no labeled CS neurons, but in infants 10 days old the gap was heavily populated, making the two bands continuous. Moreover, preliminary studies showed that some other cortical neurons outside the dorsal cortex also projected to the cord in infancy.

To study the gap neurons and others whose spinal projections did not normally persist into mature life, we made certain measures of the postnatal growth of the mantle, against which we plotted the normally developing distribution of CS neurons from which the caudal and rostral band evolve. Then we tested whether or not the gap neurons and some others would keep their spinal projections, that is, exhibit "plasticity," if other neurons in the developing caudal band were destroyed at various stages of infancy. A preliminary report and demonstration of some of the findings have been made (2).

\section{METHODS}

\section{Animals}

We used chiefly the F1 male and female offspring (Irish) of a cross between black selfed nonagouti male rats and Wistar albino females, each from lines brother-sister mated more than 70 generations, and a line of hooded rats derived from the Irish and more than 20 generations brothersister mated. Two of the albinos were used in a brain growth experiment. Housing and care of the animals were fully accredited by the American Association for Accreditation of Laboratory Animal Care. Conduction of the experiments conformed to standards in the guide for the Care and Use of Laboratory Animals, U.S. Department of Health, Education, and Welfare Publication 73-23, 1972. The animals were observed several times daily. Operations were carried out under a microscope, with the animals under ether anesthesia, in an animal surgery.

\section{Postnatal Growth of the Rat's Mantle}

The mantle and its cortex grow unevenly, not uniformly, as an expanding sphere $(3,6,7)$. The purpose of the following experiments was to 
observe how this uneven expansion of the mantle and cortex occurred and to help in making appropriate drawings on which to plot the HRP-labeled CS neurons at different ages and after injury.

Marking the Skull. Small, round marks (about 0.2 to $0.3 \mathrm{~mm}$ in diameter) were made through a surgical scalp incision on one side of the skulls of rats $1,2,5,7$, or 10 days old by making a depression in the bone with a fine drill into which surgical bone-wax blackened with India ink was inserted. (The insertion of tiny pieces of fine tungston wire through the skull into the brain surface as markers usually interfered with growth, to give unsatisfactory results.) Three rats were marked with wax at 1 day, four at 2 days, one at 5 days, nine at 7 days, and three at 10 days. The marks, $1 \mathrm{~mm}$ apart up to 7 days and $2 \mathrm{~mm}$ at 10 days, were nade in one to three parallel longitudinal rows, with the bregma and midline as references. Photographs and drawings were made at the beginning and end of the experiment. When the animals were 3 weeks [the brain is then almost adult size (7)」 to 10 weeks old, they were killed and the relative positions of the marks were compared with the original marks. Tracings of the marks in photographs or, in some instances, drawings of the skulls were transferred to outlines of brains, using the bregma and midline as references. Composite examples are shown in Fig. 1, where two experiments are shown for 2 and 7 days and one for 10 days. The assumption was made that the expanding surface of the mantle of the brain and the skull grew together, especially in the vertex region, the center of our interest. There were usually few, somtimes no, losses of marks, and the marks did not alter skull growth compared to the normal side.

Plotting Growing Cortical Areas. The growth of areas 3, 4, and 6 and adjacent regions was estimated by measuring their domains in serial frontal and sagittal paraffin-embedded microscopic sections of brains stained with luxol fast blue and cresyl violet in rats that were $2,3,5,7,10$, and 14 days and 6 and 10 weeks old. There were two rats for each age. A calibrated scale in the microscope was used. Among other histologic characteristics, areas 4 and 6 had a modestly developed layer IV (beginning to differentiate histologically in the early infant), and these areas contrasted with the cingular cortex medially, area 10 rostrally whose layer IV was scantily populated, and areas 29c, 18, and 7 caudally. Area 3 laterally was notable for its well-devcloped layer IV; its lateral border, in turn, contrasted with adjacent area 2 , whose layer IV was very densely populated with small neurons. We plotted areas 3, 4, and 6 as an aggregate (hereafter 3,4 , and 6 ) because we could not readily distinguish 4 from 6 and it was largely within the aggregate that we had located the caudal band of CS neurons in mature rats (8). Measures included those in sagittal planes through the lateral part of the habenulointerpeduncular tract, the longi- 

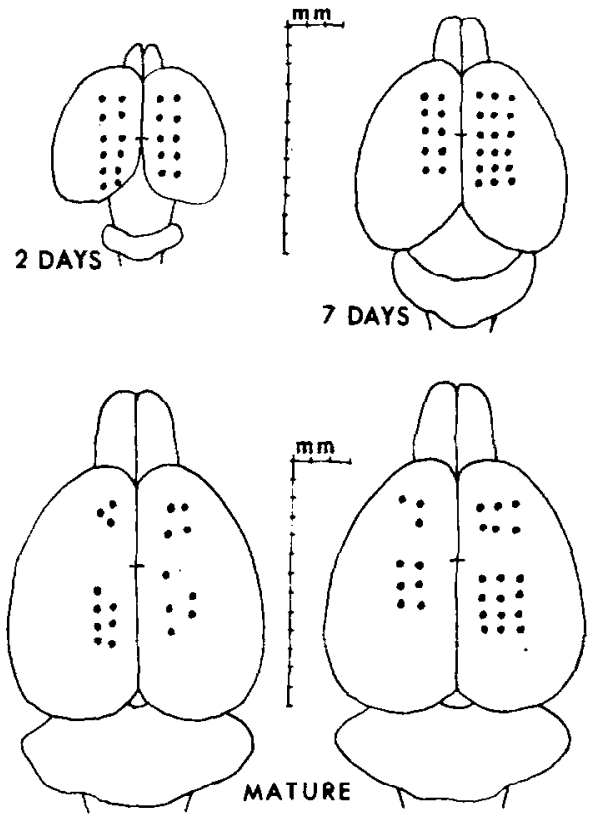
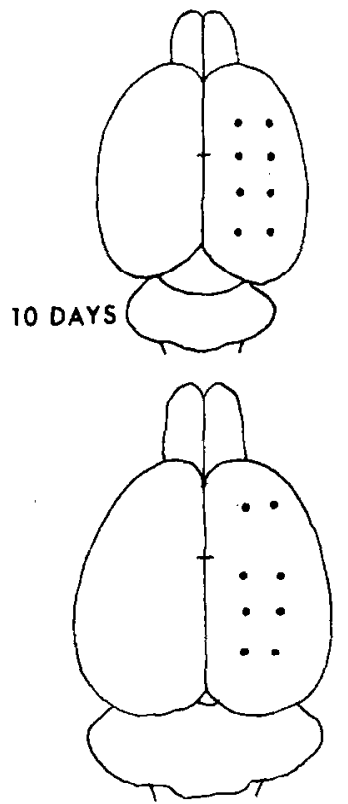

FIG. 1. Relative displacements of skull marks during development in the rat, superimposed on outlines of the brain. The upper figures represent the marks at the time they were made, and the lower figures their final positions at maturity. The short transverse line across the midline is the bregma. Greatest longitudinal growth is near the bregma and in the occiput from the 2 nd and 7 th days and near the bregma from the 10th day. Two experiments are represented at 2 and 7 days, one at 10 days.

tudinal component of the anterior commissure, and the medial geniculate. These planes were about $1,1.5$, and $2.5 \mathrm{~mm}$ from the midline in the adult rat. Measures in frontal planes included those passing rostral to the transverse portion of the anterior commissure, through the beginning of the dorsal hippocampus, and through the center of the dorsal hippocampus. These sagittal and frontal measures were transcribed to the outlines of dorsally viewed fixed brains, as shown in Fig. 2. Fixation of these brains was in $10 \%$ formalin in $0.8 \%$ sodium chloride (formol-saline), and the outlines were based on measures and tracings of two to six rats at each age.

Errors and Corrections. The difficulties of measuring the brain, especially when data from fresh specimens and specimens fixed, embedded, and sectioned in different ways must be interrelated, have frequently been noted, but never entircly surmounted $(8,9,11)$. The length of the cerebral hemisphere of our adult rats in the fresh state at autopsy was 15 to $16 \mathrm{~mm}$ and, after formol-saline fixation, 13 to $13.5 \mathrm{~mm}$. Embedded in paraffin and mounted in Permount, the length was $12 \mathrm{~mm}$, but in frozen sections 

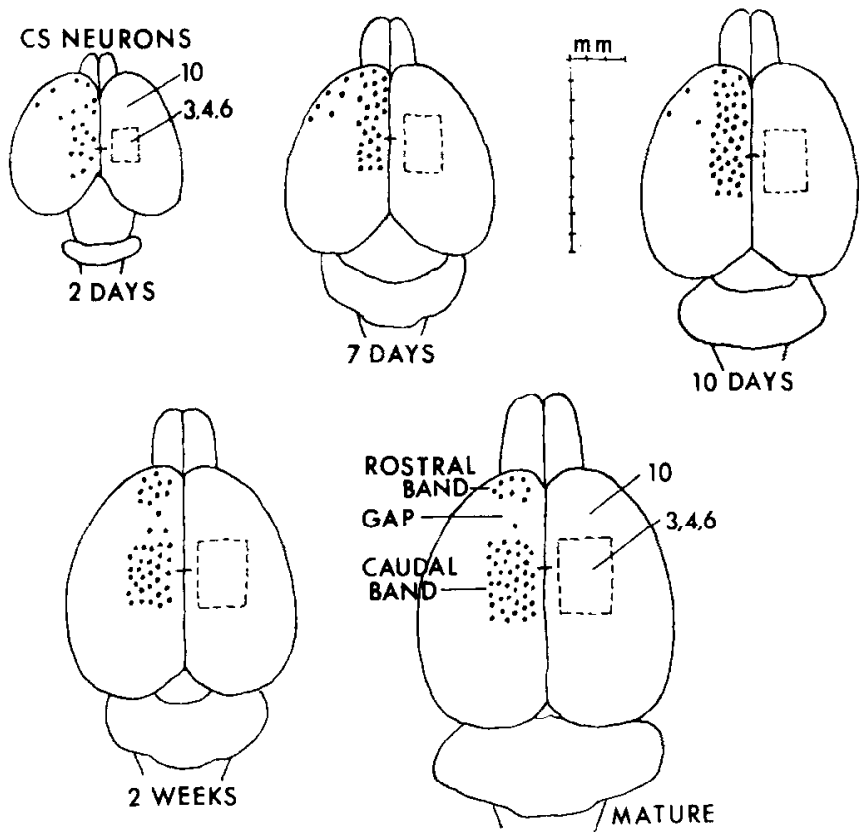

FIG. 2. The cortical areas 3,4 , and 6 , represented in aggregate as a rectangle, are shown on the right hemisphere of outlines of the brains of rats of several ages. The distribution of corticospinal neurons labeled with horseradish peroxidase from the cervical cord is represented by dots on the left. Such neurons were at first widespread, but after about 2 weeks those that could be laheled were confined to a caudal and a rostral band with a gap between. Area 10 is indicated. The short transverse line across the midline is the bregma.

dehydrated and similarly motnted for HRP studies, it was $10 \mathrm{~mm}$ (8). Some of Krieg's areas have sharp borders, others have not, and an "error" to $0.5 \mathrm{~mm}$ or more was possible in deciding where to draw some lines. The skull marks and measures of area 3,4 , and 6 were transcribed directly to the fixed brain outline without corrections (which were very small), because the relative position of the marks was the point being marle and areas 3, 4, and 6 were approximations, as indicated. The positions of HRP-labeled neurons, described later, were transcribed from their regional positions in sections to corresponding regions in the outlines shown in Figs. 2 and 5, the purpose being to show their changing distribution during postnatal life and after injury.

\section{Horseradish Peroxidase Methods}

These were described in detail previously (8). Essentially they consisted of surgically incising the dorsal spinal cord transversely, with very fine 
scissors under a microscope, and introducing HRP crystals directly into the wound. The rat has one of the largest CS tracts in proportion to brain size among mammals; the tracts form the medullary pyramids and completely decussate caudally, to run in the ventral part of the dorsal columns of the spinal cord. The cord incisions included the dorsal columns and various adjacent structures. During the period between insertion of the HRP and killing of the animals for histologic study, infant animals were kept at about $29^{\circ} \mathrm{C}$ and maintained by feeding a mixture of cream, saline, and glucose solutions and by subcutaneous injections of saline solution with glucosc. Mature animals were given regular food and watcr and supplemental subcutaneous saline solution with glucose if necessary.

Three normal rats were studied at 2 days of age, one rat on the 4 th day, one on the 5th day, seven on the 7th day, eight on the 10th day, and two each on the 13th, 18th, 21st, and 25th days. Three of the 7-day rats received $\mathrm{HRP}$ at the first lumbar level (L1) and four at the first cervical level (C1). Four 10-day rats received HRP at $\mathrm{C} 1$ and four at L1. In all experiments, the brain with the first cervical segment of the cord attached was divided sagittally; usually one half was serially sectioned sagittally and the forebrain of the other half was cut frontally, but sometimes both halves were cut sagittally. Sections were collected successively in five jars. The sections in at least one jar, and usually two, were prepared for demonstration of HRP by dark- or light-field microscopy, using diaminobenzidine or benzidine counterstained with Darrow red.

\section{Ablation of Cortex in Infancy}

Cortex was aspirated by a blunt 27 - to 30 -gauge hypodermic needle through a surgical skull opening under a microscope, with the animal under ether anesthesia as detailed previously (8). In preliminary experiments the whole motor-sensory cortex, which included the domains of the caudal and rostral bands (8), was removed on one side in five rats 1 or 2 days old. The purpose was to see whether or not this affected the development of the opposite gap C.S nelrons. In more extensive experiments, the domain of the caudal band, corresponding approximately to areas 3,4 , and 6 , was removed bilaterally in rats of various ages. Three rats 2 days old, two rats 5 days old, three rats 7 days old, and three rats 10 days old had this operation. An additional two rats 5 days old had one caudal band removed. All these rats then had HRP introduced into the cord at $\mathrm{C} 1$ when they matured, as in the normals, except for one 7 days old and one 10 days old, whose HRP was introduced at L1. Serial sections of the brains were prepared for demonstration of HRP as in the normal controls. 


\section{RESULTS}

\section{Postnatal Growth of the Rat's Mantle}

Marking the Skull. Earlier work showed that the distance from nasion to bregma grew from 3.5 to $4 \mathrm{~mm}$ between 1 and 2 days and to 5 or 5.5 $\mathrm{mm}$ by 3 weeks; the distance from bregma to lambda increased from about 4 to $8 \mathrm{~mm}$ between 1 day and 2 weeks (7). The present experiments, represented in Fig. 1, showed that within these broadly defined regions there were areas of greater and lesser expansion. Beginning at 1 to 2 days of age, the greatest longitudinal expansion of the dorsal skull was in the occipital region and in the region near the bregma. Lesser longitudinal expansion occurred in the other regions, for example, near the frontal pole, and there was some lateral expansion within the vertex, as we previously showed by autoradiographic cell labeling and radiation studies $(3,5,6)$. When growth was measured from the 7 th day, it continued to be substantial in the occipital and bregma regions and less in the other regions. When the measures were begun on the 10th day, substantial longitudinal expansion had continued near the bregma region.

Plotting Growing Cortical Areas. These experiments are represented in Fig. 2, showing the expanding areas 3, 4, and 6. (The distribution of HRP-labeled CS neurons, discussed in the next section, are also shown.) This expansion was a reflection of the progressive differentiation of the neurons in the dorsal cortex: maturation of their nuclei, cytoplasma, and apical dendrites, coupled with the arrangement of the cells into layers, contributing to the formation of the cytoarchitectural regions. There was a general progression of this form of differentiation from rostral to caudal parts of the dorsal cortex in the first week, when neurons, especially of layers IV, III, and II, were substantially less differentiated in the occipital than in the dorsal frontal regions. We had not observed this front to back gradient before, but in previous work we showed a gradient extending from the lateral isocortex medially to the vertex in respect both to cell migrations that formed the cortex pre- and postnatally and in the ensuing differentiation of the neurons. [For example, many of the large neurons in layer $\mathrm{V}$ of the lateral isocortex, some belonging to the corticospinal system and projecting to the medulla, originated about the 15 th prenatal day and arrived at their cortical destinations within 2 to 3 days. Many comparable neurons in the dorsal cortex did not originate until the 17 th day and took several days to arrive at their places in the cortex (6). Further histologic differentiation showed a similar lag between the two regions $(1,4)$.] Cell migrations and differentiation in the frontal pole and occipital region paralleled the vertex, lagging behind the more lateral regions. 
A comparison of Figs. 1 and 2 shows that differentiation of 3, 4, and 6 into recognizable cytoarchitectural areas progressed not only in the region of great expansion around the bregma, but also in the region behind it, where expansion was relatively less. Expanding growth of any part of the mantle was the product of several factors: postnatal migrations of neurons to the outer cortex and of neuroglia into the isocortex generally, as well as the ingrowth of corticopetal fibcrs and the phenomena of ccllular and cytoarchitectural differentiation that we mentioned above. We did not try to find precise correlations between rates of cellular differentiation in parts of the caudal band and rates of expansion in different parts of the mantle. However, the general relations were that, by 2 weeks, expansive growth of the mantle was subsiding, areas 3, 4, and 6 were nearing adult size, and the caudal and rostral bands, as detailed below, had been largely defined.

\section{Normally Developing CS Neurons Labeled with HRP}

CS neurons labeled from the cervical cord at different ages, that is, all CS neurons whose axons extended to that level or further at the time, are represented diagrammatically in Fig. 2. Normal CS neurons labeled with HRP in rats 7 days old are photographed in Figs. 3 and 4. In the infant,

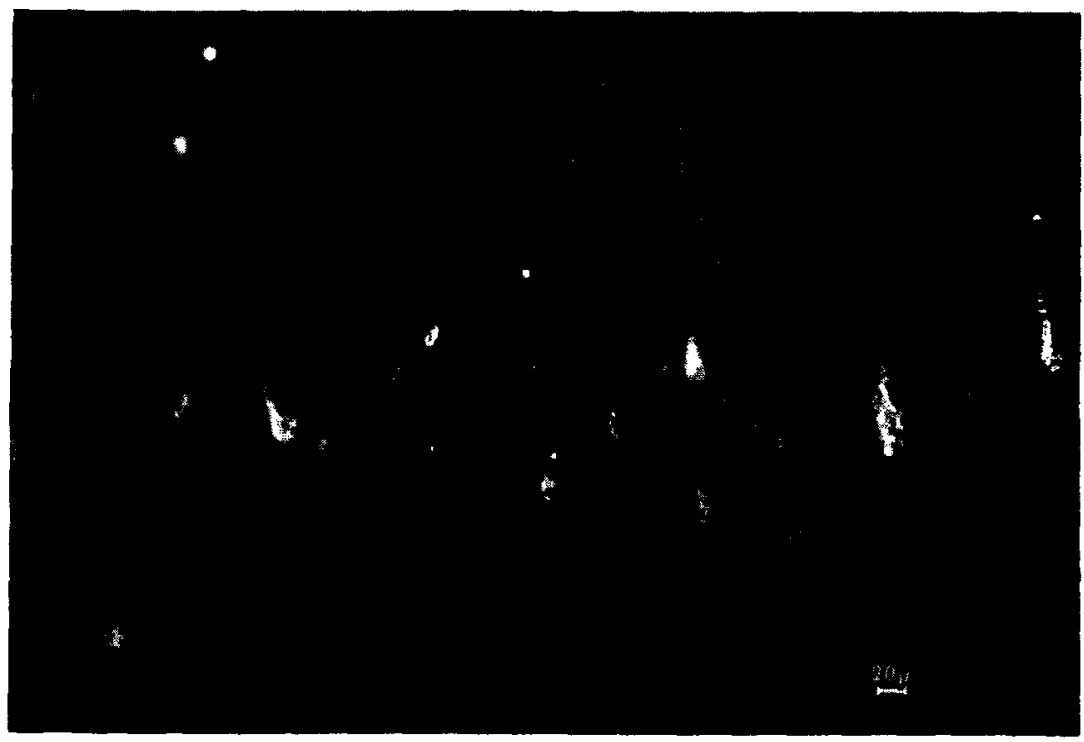

FIa. 3. Corticospinal neurons in layer $V$ of the dorsal isocortex in the gap region of a normal rat 7 days old, labeled with horseradish peroxidase from the cervical cord. At this age, the neurons often show prominent labeling of their apical dendrites. Two heavily labeled neurons appear almost superimposed on the right. Dark field, diaminobenzidine. 


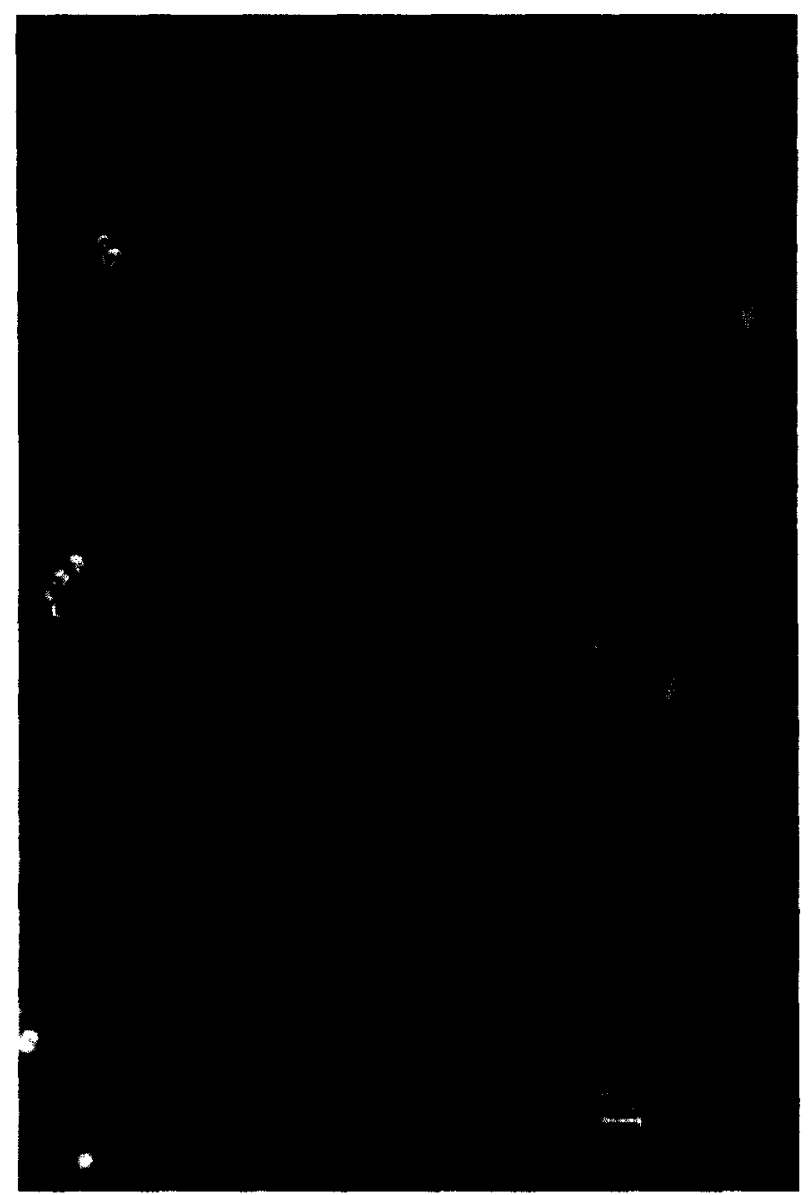

FIG. 4. Two corticospinal neurons in the far medial cingular cortex of a normal rat 7 days old, labeled with horseradish peroxidase from the cervical cord. They are principally visible by their apical dendrites. The medial vertical surface of the cerebral hemisphere appears at the left for orientation. Dark ficld, diaminobenzidinc.

especially during the first week, apical dendrites were proninently labeled. These neurons, all in layer $V$, were most widespread in the cortex in the infants 4, 5, 7, and 10 days old. In this period, they were not only present in the domains of the caudal and rostral bands, but were also numerous in the gap region between them, as shown in Fig. 3. A few were found very far medially in the cingular cortex, their long axes lying almost in a horizontal plane, as shown in Fig. 4, and at comparable extremes in the lateral isocortex and the frontal pole. By about 2 weeks (13 days), these extraneous neurons could no longer be labeled from the cervical spinal cord, except for a few more in the gap than would be seen in the adult. 


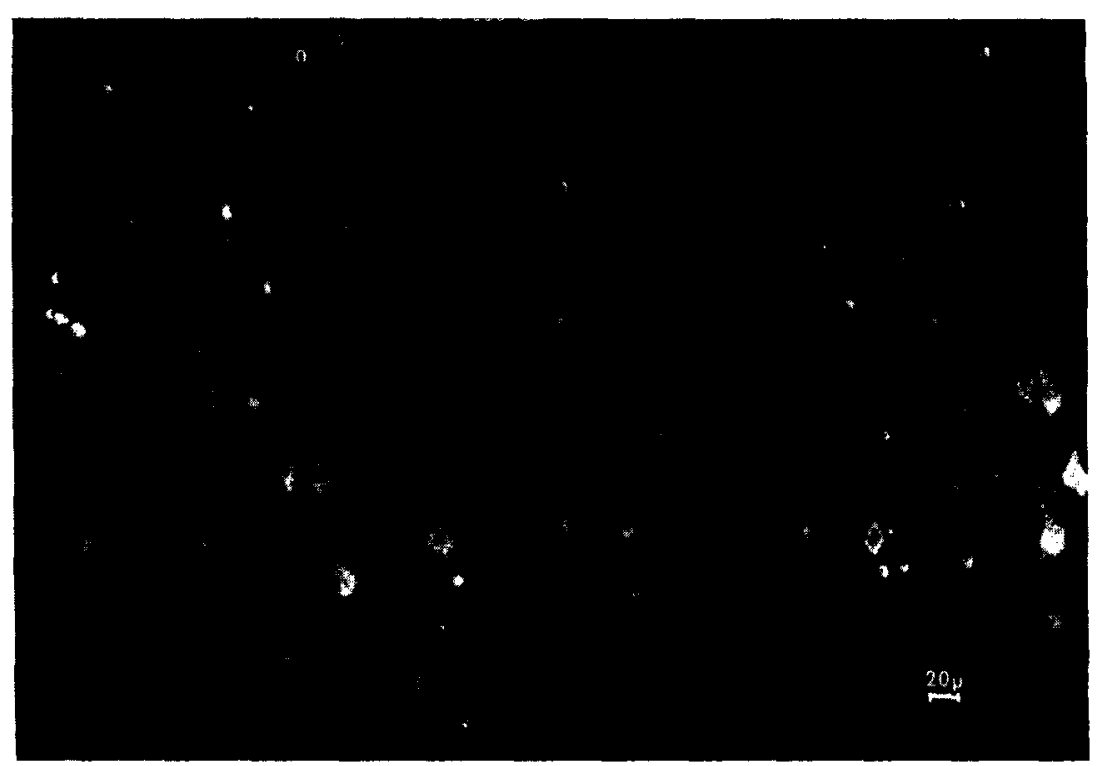

FIG. 5. Corticospinal neurons in layer $\mathrm{V}$ of the dorsal isocortex of the gap region of a mature rat whose caudal bands were largely ablated at 10 days of age. They were labeled with horseradish peroxidase from the cervical cord. Normally very few or no such neurons are seen here. Dark field, diaminobenzidine.

After this, the adult picture of separate caudal and rostral bands, with rare or no labeled neurons in the gap between them, was attained.

The disappearance of the gap neurons and other extraneous CS neurons occurred within 3 or 4 days. We saw virtually no necrosis of cortical neurons in an extensive series of rats in this period of 10 to 14 days, although occasional necrotic neurons were seen in very early infancy.

When labeling was done from the L1 level, no extraneous neurons were seen in the gap or elsewhere. By 7 days, CS neurons could be demonstrated throughout the domain of the caudal band by labeling from L1. This was in accord with our earlier fiber degeneration and tritiated amino- acid autoradiographic studies on the growth of the CS tract showing that some $\mathrm{CS}$ axons had reached the lumbar cord toward the end of the first week (7). An additional observation was that axons from neurons in the red nucleus, medullary reticular formation, and lateral vestibular nucleus had reached the lumbar cord by 7 days.

\section{Ablation of Cortex in Infancy}

When one motor-sensory cortex was ablated at 1 or 2 days of age, there was a persistence of a few CS neurons in the gap on the uninjured side into mature life, demonstrated when HRP was introduced at the C1 level. If the caudal band region (essentially developing areas 3, 4, and 6) 

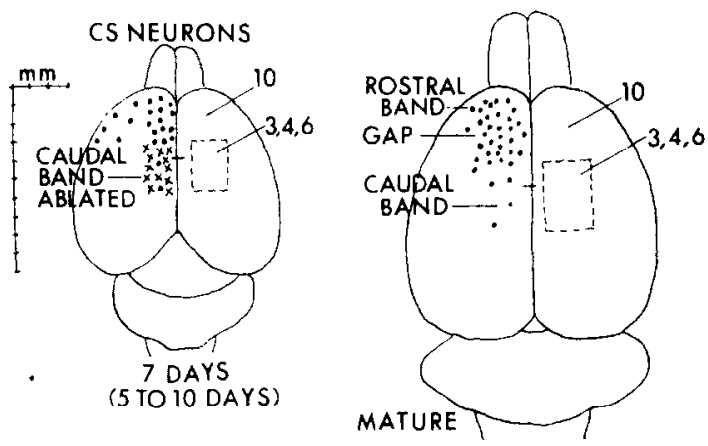

FIG. 6. The distribution of corticospinal neurons labeled with horseradish peroxidase is represented by dots on the left hemisphere of the outlines of brains of 7-day-old and mature rats. The caudal band was ablated at 7 days of age, as indicated in the left figure. Cortical areas are shown on the right, as in Fig. 2. Corticospinal neurons persisted in the gap region when the caudal band was ablated between 5 and 10 days of age.

was removed bilaterally at 2 days, the results were similar. However, when the caudal band region was bilaterally removed at 5,7 , or 10 days, the results were impressive: Substantial numbers of CS neurons were demonstrated in the gap (Fig. 5), forming a band of CS neurons in the gap continunus with the rostral hand (Fig. 6). When the caudal band region was ablated unilaterally at 5 days, CS neurons were demonstrated in the gap on both sides, as in the bilateral ablation experiments. In the two experiments where labeling was done from the L1 level, no labeled cells appeared in the gap, showing that the axons of the gap CS neurons did not extend that far. The ablations usually spared some CS neurons in the caudal band, showing that total destruction of this population of neurons was not necessary to stimulate persistence of gap CS neurons.

\section{DISCTUSSION}

Measurements of the differential expansion of the developing mantle, the histologic maturation of the cytoarchitectural areas, and the growth of corticospinal neuron axons did not parallel each other in simple ways. We noted under Results that individual neuron growth, cell migrations, and influx of corticopetal fibers were going on in various combinations of rates, thus contributing to the complexity. Especially interesting was the observation that axoms of $C S$ neurons had advanced through the brain to the cord, a form of differentiation, when their cell bodies were still fairly immature, whereas the cytoarchitectural regions from which they originated were just becoming recognizable.

The formation of transient axon projections to the cord by neurons in the gap and elsewhere may have been analogous to the well-known forma- 
tion of exploratory neurites in the developing nervous system of lower forms. For example, in the chick, the number of spinal motor neurons that was formed exceeded that needed for muscle innervation, and in the competition for peripheral targets, those that did not make permanent connections died. There was no evidence that the gap neurons died, and we assumed that their transient projections atrophied or were withdrawn. When competition from caudal band neurons was removed by ablation, they were able to keep their CS projections.

The programming of the growth of both transient and permanent CS axons is little understood. We showed earlier with fiber degeneration and autoradiographic methods that CS axons passed the cervical region soon after birth and reached the lumbar level at 5 to 7 days of age (7). We traced these early axons to the developing caudal band region, and if that region was ablated in the first few days after birth, CS neurons in the contralateral caudal band region sent axons into the empty CS pathway, forming a small uncrossed CS tract. However, these aberrant fibers did not descend through the medulla to the cervical cord until the third week, along with the majority of CS axons that was then growing caudalward. The aberrant fibers were stable and could be identified for at least 16 months, but whether the early fibers to the lumbar region, which may have been pilots to establish the CS tracts, were permanent or transient is not known.

To test the functions, if any, of the CS gap neurons that persisted after caudal band ablation, we began ablating the gap region in normal mature animals and in mature animals whose caudal bands were ablated at 7 days, then observing their locomotion on difficult pathways and their placing reactions. Ablation of the frontal cortex, including the gap region but sparing the caudal band, had not previously impaired (7), and did not in these animals impair, locomotion or placing unless it encroached on the caudal band behind it. Removing the gap in mature animals whose caudal bands were ablated in infancy did not appreciably worsen or lessen the impairment of locomotion that the infant lesion produced, as far as the studies have progressed. Animals with one caudal band ablated in infancy, which it will be recalled retain their gap CS neurons on both sides, may give us more information when the contralateral gap is ablated at maturity.

\section{REFERENCES}

1. D'Amato, C. J., ANd S. P. Hicks. 1965. Effects of low levels of ionizing radiation on the developing cerebral cortex of the rat. Neurology 15 : 1104-1116.

2. D'A мato, C. J., ANd S. P. Hicks. 1977. Normal and post-traumatic development of corticospinal neurons studied with horseradish peroxidase. Neurosci. Abstr. $3: 66$. 
3. Hicks, S. P. 1958. Radiation as an experimental tool in mammalian developmental neurology. Physiol. Rev. $38: 337-356$.

4. Hicks, S. P., M. C. Cavanagh, and E. D. O'Bkikn. 1962. Effects of anoxia on the developing cerebral cortex in the rat. Am. J. Pathol. 40:615-635.

5. Hicks, S. P., and C. J. D'Amato. 1961. How to design and build abnormal brains using radiation during development. Pages $60-97$ in W. S. FieldS AND M. M. Desmond, Eds., Disorders of the Developing Nervous System. Thomas, Springfield, Ill.

6. Hicks, S. P., and C. J. D'Amato. 1968. Cell migrations to the isocortex in the rat. Anat. Rec. $160: 619-634$.

7. Hicks, S. P., and C. J. D'Amato. 1975. Motor-sensory cortex-corticospinal system and developing locomotion and placing in rats. Am. J. Anat. 143: 1-42.

8. Hicks, S. P., ANd C. J. D'Amato. 1977. Locating corticospinal neurons by retrograde axonal transport of horseradish peroxidase. Exp. Neurol. 56: 410420.

9. König, F, R., And R. A. Klippel. 1963. The Rat Brain: A Stcreotaxic Atias. Williams and Wilkins, Baltimore.

10. Krifg, W. J. S. 1946. Connections of the cerebral cortex. I. The albino rat. A. Topography of the cortical areas. B. Structure of the cortical areas. J. Comp. Neurol. $84: 221-323$.

11. Kriki, W. J. S. 1946. Accurate placement of minute lesions in the brain of the albino rat. (.). Bull. Northwest. Univ. Med. School $20: 199-208$. 